\title{
Étienne GUILLAUD, De l'attrait à l'usure. Les trajectoires professionnelles des éducateurs sportifs en nautisme
}

Thèse de doctorat en sociologie, Université de Nantes, dirigée par Annie Dussuet et Gildas Loirand (soutenue le 10 décembre 2018)

From attraction to professional wear. Professional trajectories of sports teachers in nautical activities

\section{Étienne Guillaud}

\section{OpenEdition}

\section{Journals}

Édition électronique

URL : http://journals.openedition.org/tourisme/2479

DOI : 10.4000/tourisme.2479

ISSN : 2492-7503

Éditeur

Éditions touristiques européennes

Référence électronique

Étienne Guillaud, «Étienne guilıaud, De l'attrait à l'usure. Les trajectoires professionnelles des éducateurs sportifs en nautisme », Mondes du Tourisme [En ligne], 16 | 2019, mis en ligne le 01 décembre 2019, consulté le 23 septembre 2020. URL : http://journals.openedition.org/tourisme/2479 ; DOI : https://doi.org/10.4000/tourisme.2479

Ce document a été généré automatiquement le 23 septembre 2020.

\section{c) $(1) \ominus$}

Mondes du tourisme est mis à disposition selon les termes de la licence Creative Commons Attribution - Pas d'Utilisation Commerciale - Pas de Modification 4.0 International. 


\section{Étienne GUILLAUD, De l'attrait à l'usure. Les trajectoires professionnelles des éducateurs sportifs en nautisme}

Thèse de doctorat en sociologie, Université de Nantes, dirigée par Annie Dussuet et Gildas Loirand (soutenue le 10 décembre 2018)

From attraction to professional wear. Professional trajectories of sports teachers in nautical activities

Étienne Guillaud

1 Cette thèse interroge les conditions de production du sentiment d'usure propre aux éducateurs sportifs en nautisme, entendus comme les titulaires d'une certification - un brevet d'état (BE) ou un BPJEPS - leur permettant d'encadrer contre rémunération tout au long de l'année les activités de voile, char à voile, kayak, surf et kitesurf. L'usure au sein de cet univers professionnel, caractérisé par un fort engagement des travailleurs, n'est jamais indépendante des attraits ayant présidé à l'entrée dans le métier. Elle peut finalement être définie comme le processus de dévaluation de ces attraits au cours de la trajectoire professionnelle. Une soixantaine d'entretiens et des observations dans divers établissements nautiques du littoral de la région Pays de la Loire constituent le cœur du travail d'enquête, mené entre 2013 et 2017. Le développement des activités nautiques en Pays de la Loire, historiquement moins implantées que dans d'autres régions (comme la Bretagne), est un enjeu politique fort en matière de valorisation touristique. Mais les conditions de travail et d'emploi des travailleurs, qui permettent ce développement, demeurent peu visibles.

2 Ainsi, le travail d'éducateur sportif en nautisme est souvent présenté sur le mode d'un «métier-passion », voire d'une " chance ", pour son cadre d'exercice (le bord de mer), les bonnes relations entretenues au sein des établissements ou encore le caractère gratifiant de la transmission des activités nautiques. Or, derrière cette image 
valorisante, il s'avère que les départs du métier sont fréquents après quelques années d'exercice au point que ce turnover pose problème au sein des établissements nautiques. En effet, l'exercice du métier est marqué par une pluralité de difficultés, comme des rémunérations relativement faibles, des effets de saisonnalité (Guibert et Slimani, 2011) ou bien encore un coût physique exigeant durant les phases d'encadrement.

\section{L'espace professionnel de l'encadrement du nautisme}

3 Au-delà du caractère peu visible des difficultés au travail, un autre trait singulier du métier de service d'éducateur sportif est de se situer à l'intersection du " marchand » et du «non-marchand». S'ils peuvent assurer des prestations socio-éducatives ou sportives considérées comme des activités reconnues d'utilité publique ou sociale, les centres nautiques du littoral assurent une large part de leur chiffre d'affaires par le biais de prestations commerciales, souvent plus répétitives et techniquement moins intéressantes, principalement à destination des touristes durant la période estivale. La nécessité de commercialiser leurs prestations pour la survie économique de leurs établissements obligent alors les éducateurs sportifs à devenir des «agents de développement» des activités nautiques. Les communes littorales bénéficient de ce travail, qui contribue à faire du nautisme une ressource territoriale pour elles. Mais cela ne se traduit pas en retour par la reconnaissance de ce travail ou par une contribution à l'amélioration des conditions d'emploi de ces travailleurs de la part de ces communes.

4 L'encadrement des activités nautiques correspond aux tâches effectuées pour et sur les usagers (Hughes, 1996) : il est considéré comme le cœur de l'activité et reste son aspect le plus visible. Il engage la maîtrise de compétences relationnelles (pour assurer la transmission de savoirs et savoir-faire), mais aussi un sens pratique de l'encadrement (pour assurer la sécurité). L'encadrement ne peut toutefois résumer l'ensemble des tâches des éducateurs sportifs en nautisme. D'autres tâches annexes sont importantes : la gestion et la réparation du matériel, la gestion administrative et financière, le management des équipes saisonnières, les activités de formation... Ces tâches peuvent s'avérer convoitées, notamment avec l'âge, à la fois pour éviter la lassitude en diversifiant ses tâches mais aussi pour protéger sa santé en allant moins souvent « sur l'eau ».

5 La répartition des tâches et des prestations devient dès lors l'objet de rapports de pouvoir, souvent déniés comme tels, au sein des établissements nautiques. Le groupe professionnel peut ainsi s'envisager comme un champ (Quijoux, 2015), au sein duquel les éducateurs permanents (en CDI ou titulaires de la fonction publique) tendent à s'attribuer les publics et les tâches les plus valorisés et les plus à même de les protéger des difficultés au travail au dépend des saisonniers (en contrat de 5 à 8 mois). La concurrence pour l'obtention des postes stables est forte depuis le milieu des années 2000 et place les saisonniers dans des situations de «loyautés incertaines " (Jounin, 2009). L'enjeu du champ est de bénéficier d'une reconnaissance en tant que "professionnel». Cette reconnaissance passe par des jugements professionnels (compétence relationnelle avec l'usager, maîtrise du sens pratique, connaissances techniques...) et moraux (bonne hygiène de vie, capacité à "prendre sur soi » ...) circulant entre éducateurs stabilisés dans l'emploi et en mesure d'assurer les recrutements et les réputations des uns et des autres. La constitution d'un capital 
symbolique pour se faire reconnaître au sein du champ peut ainsi déterminer le maintien ou non dans l'emploi. Toutefois, les éducateurs sportifs en nautisme doivent aussi mobiliser d'autres ressources en dehors de l'espace professionnel, par exemple des ressources matérielles ou économiques familiales, pour avoir des chances de perdurer dans le métier.

\section{Du reclassement au vécu de l'usure : les trajectoires des éducateurs}

6 L'orientation vers le métier d'éducateur sportif en nautisme est marquée, au départ, par une situation de frustration ou d'échec : impossibilité de trouver un emploi, vécu de l'ennui au travail, conflit avec des collègues ou employeurs, sentiment de s'être trompé d'orientation scolaire et professionnelle, etc. Ces expériences poussent les individus à se sentir déclassés et à se réorienter professionnellement. Devenir éducateur sportif n'est ainsi que rarement un premier choix et prend plutôt la forme d'une reconversion à un moment où les enquêtés voulaient « changer de vie ». Le fait d'exercer ce métier valorisant, notamment car il s'exerce en bord de mer, permet aux éducateurs de constituer leur travail comme distinctif, au sens où il n'est pas un " travail ordinaire " et limité à la seule dimension professionnelle («c'est plus qu'un travail»), ce qui leur permet de conjurer le déclassement initial. La rhétorique de "la passion" dans le groupe professionnel sert alors moins à décrire un attrait pour le nautisme ou le travail lui-même qu'à tirer un profit distinctif de soi à soi (Lahire, 2004), en se singularisant en tant que travailleur éloigné du modèle classique du salariat, et à légitimer une inscription au sein d'un espace professionnel dans lequel le dévouement "passionné » est implicitement exigé.

7 L'usure se traduit alors, pour ces éducateurs, par les difficultés vécues lorsque leur statut de travailleur "ordinaire », et même plus : de travailleur " mal rémunéré » et "mal considéré », leur est rappelé. Ces rappels peuvent survenir lors de mésentente avec leur employeur, lorsque leur rémunération ou la saisonnalité de leur emploi pose des difficultés dans la vie familiale, ou bien encore lorsque leur corps se fatigue ou se blesse. C'est le plus souvent dans l'articulation de plusieurs de ces facteurs que les éducateurs sportifs vivent, au fil du temps, une série de désajustements qui les conduisent peu à peu à percevoir leur travail comme difficile, voire à ne plus le supporter au point de devoir en sortir.

8 L'usure était, au départ de ce travail, une catégorie indigène, exprimant un sentiment diffus. En la redéfinissant comme la dévaluation des attraits initiaux pour le métier, il est possible d'en rendre compte sociologiquement et de l'extraire des explications trop "psychologisantes» ou "pathologisantes». Le processus d'usure interroge alors l'ambivalence entre visibilisation et invisibilisation du travail (Krinsky et Simonet, 2012). Cette ambivalence vient du fait que, pour se préserver de l'usure, les éducateurs sportifs en nautisme doivent tout à la fois mettre à distance le travail en tant qu'activité de travail pour en conserver la dimension distinctive et, dans le même temps, trouver les moyens d'en assurer la reconnaissance pour qu'il ne puisse faire l'objet d'une dévaluation en vieillissant. Les chances de trouver une forme d'équilibre propice à maintenir l'engagement dans le métier sont inégales et semblent plutôt bénéficier à ceux qui sont déjà les plus disposés, par leurs positions et leurs ressources, à accéder à des formes de reconnaissance professionnelle. 


\section{BIBLIOGRAPHIE}

Christophe GUIBERT et Hassen SLIMANI, Emplois sportifs et saisonnalités. L'économie des activités nautiques : enjeux de cohésion sociale, L'Harmattan, 2011.

Everett C. HUGHES, Le regard sociologique. Essais choisis, Éditions de l'EHESS, 1996.

Nicolas JoUnIN, Chantier interdit au public. Enquête parmi les travailleurs du bâtiment, La Découverte, 2009.

John KRINSKY et Maud SIMONET, « Déni de travail : l'invisibilisation du travail aujourd'hui. Introduction ", Sociétés contemporaines, $\mathrm{n}^{\circ}$ 87, 2012.

Bernard LAHIRE, La culture des individus. Dissonances culturelles et distinction de soi, La Découverte, 2004.

Maxime QUIJoux, « Première partie. Bourdieu et le travail, une introduction », in Maxime QujJouX (dir.), Bourdieu et le travail, Presses Universitaires de Rennes, 2015.

\section{AUTEUR}

\section{ÉTIENNE GUILLAUD}

etienne.guillaud@univ-nantes.fr 